\title{
Post-embryonic pericardial cells of Drosophila are required for overcoming toxic stress but not for cardiac function or adult development
}

\author{
Debjani Das • Rajaguru Aradhya - D. Ashoka • \\ Maneesha Inamdar
}

Received: 29 June 2007 / Accepted: 6 September 2007 / Published online: 7 November 2007

(C) Springer-Verlag 2007

\begin{abstract}
The Drosophila heart is composed of two cell types: cardioblasts (CB) and pericardial cells (PC). Whereas CBs act to maintain rhythmic contractions, the functions of accessory PCs are not clear. The close association between these two cell types has led to speculation of a cardioregulatory role for PCs. However, we find that viability and cardiac function are normal in larvae following post-embryonic ablation of PCs by induced cell death. Removal of PCs during the larval instars or before metamorphosis results in viable and fertile adults. Interestingly, such animals have a reduced lifespan and increased sensitivity to toxic chemicals. Thus, although PCs may have an embryonic role in cardiogenesis, they do not appear to play a part later in cardiac function as suggested. However, the role of PCs in the uptake and sequestering of toxins, their sensitivity to toxic stress and the decreased lifespan of animals without PCs indicate the importance of PCs in organismal homeostasis.
\end{abstract}

Keywords Pericardial cell $\cdot$ Heartbeat . Cardiac remodelling · Lifespan · Toxic stress .

Drosophila melanogaster (Insecta)

\section{Introduction}

The Drosophila heart (or dorsal vessel) is a linear unbranched structure consisting of a contractile tube of

D.D. is supported by a CSIR-SPM fellowship. This work was funded by the JNCASR, the Council of Scientific and Industrial Research, and the Department of Biotechnology, Government of India.

D. Das $\cdot$ R. Aradhya $\cdot$ D. Ashoka $\cdot$ M. Inamdar $(\bowtie)$

Molecular Biology and Genetics Unit, Jawaharlal Nehru Centre for Advanced Scientific Research,

Jakkur P.O., Bangalore 560064, India

e-mail: inamdar@jncasr.ac.in cardioblasts (CBs) flanked by accessory pericardial cells (PCs). Studies on heart development have identified molecular players and regulatory networks that later have proved to be essential, even for vertebrate cardiogenesis. Hence, the Drosophila heart has been a model of choice for investigating molecular regulation of early cardiac development (Cripps and Olson 2002). This simple structure with few cellular subtypes has also proved to be a highly informative model for human cardiac diseases such as myopathies, channelopathies and age-related cardiac dysfunction (Bier and Bodmer 2004). Among the two dorsal vessel cell types, the direct role of the CBs in rhythmic contraction is well established and studied (Monier et al. 2005). However, PC function is less clearly understood. Because of their close association with CBs, PCs have long been implicated in cardiac regulation. This appears to be true, at least during embryonic development, since embryos with altered PC number and/or PC-CB association fail to complete cardiogenesis (Alvarez et al. 2003; Chartier et al. 2002; Yi et al. 2006). Nevertheless, the requirement of PCs, if any, for cardiac function has not been thoroughly investigated.

Two recent studies involving alteration to the PC number at the embryonic stage and scrutiny of subsequent postembryonic cardiac function suggest an association between PC number and post-embryonic cardiac function (Fujioka et al. 2005; Johnson et al. 2007). Since PCs are known to be required for normal cardiogenesis, this raises an important concern about the contribution of developmental defects to the observed cardiac malfunction. This view is supported by the hypothesis put forward by Johnson et al. (2007) to explain the effect of increased PC numbers on post-embryonic cardiac output: excessive deposition of the extracellular matrix protein Pericardin $(\mathrm{Prc})$ around the cardiac tube during cardiogenesis limits the diastole volume 
thereby reducing cardiac output. Thus, unperturbed dorsal vessel development becomes a prerequisite for any attempt to clarify the cardio-regulatory role, if any, of PCs. We have used apoptosis-mediated ablation of PCs as a method to investigate their post-embryonic role. Using the TARGET (McGuire et al. 2003) system for tight spatial and temporal control, we have ablated PCs after the completion of dorsal vessel development. This strategy has allowed us to correlate the phenotypes observed with PC function without raising concerns about developmental defects. We report that ablation of all PCs post-embryogenesis does not affect adult development or fertility. Complete absence of post-embryonic PCs has no effect on heart rate but flies lacking PCs have a significantly shorter lifespan under laboratory conditions. The lifespan is reduced further under toxic stress.

\section{Materials and methods}

Ablation of PCs post-embryogenesis srpHemo-GAL4 UASEGFP (II) \#1643 B flies were crossed to UAS-hid/CyO GFP; gal $80^{\text {ts }}$ flies at $18^{\circ} \mathrm{C}$. The embryos were collected and allowed to hatch. Mid-second-instar larvae (mid-L2) were sorted based on ubiquitous and strong green fluorescent protein (GFP) expression resulting from a single copy of $C y O$ GFP. No detectable GFP fluorescence in PCs is seen in the presence of a single copy of srpHemo-GAL4 UASEGFP. Hence, all GFP-expressing larvae were considered to be of the control PC+ genotype [srpHemo-GAL4 UASEGFP/CyO GFP; tub-gal80 $\left.{ }^{t s} /+\right]$. Both GFP (srpHemoGAL4 UAS-EGFP/CyO GFP; tubgal80 $0^{\text {ts }} /+$ ) and non-GFP (srpHemo-GAL4 UAS-EGFP/UAS-hid; tub-gal80 ${ }^{t s} /+$ ) larvae (100 in each group) were subsequently maintained at $25^{\circ} \mathrm{C}$.

Heart-rate measurements Heart-rate measurements were performed at $25^{\circ} \mathrm{C}$ on white pre-pupae by viewing them through an inverted compound microscope (Olympus IX70) to visualize the heart through the cuticle. All animals were allowed to acclimatize for $20 \mathrm{~min}$ before being counted. Heartbeats were counted for intervals of $15 \mathrm{~s}$. Individual values are given as the average of 12 continuous counts expressed in beats per second. At least 30 animals were counted for each genotype.

\section{Results and discussion}

Drosophila adults can develop without PCs

Ablating a structure often gives useful insights into its function in the physiological context. We used this strategy by employing the GAL4-UAS system (Brand and Perrimon
1993) to analyse the way that loss of PCs might affect Drosophila melanogaster. We ablated PCs by mis-expressing the pro-apoptotic gene head involution defective (hid) by using UAS-hid. To express UAS-hid only in postembryonic PCs, we required a GAL4 driver that expressed specifically in PC post-embryogenesis. We therefore examined the expression pattern of candidate genes and found that Serpent (Srp), a GATA factor well-studied for its role in haematopoiesis, was expressed in post-embryonic PCs and in haemocytes and fat bodies (Brodu et al. 1999; Mandal et al. 2004). Bruckner et al. (2004) have generated a srpHemo-GAL4 driver for analysing function in embryonic haemocytes. We characterized the post-embryonic expression pattern of this driver by using a $U A S-G F P$ reporter construct. GFP expression was seen in PCs from second larval instar onwards (Fig. 1), throughout pupal development, and in adults (not shown). We therefore used the srpHemo-GAL4 driver to restrict Hid expression to postembryonic stages and a temperature-sensitive tub-Gal80 ${ }^{t s}$ construct (a suppressor of GAL4 function) to achieve temporal control of Hid expression. Both control (wildtype) and experimental (Hid misexpressing) larvae were given continuous heat shock $\left(25^{\circ} \mathrm{C}\right)$ from mid-L2 onwards (see above). Forty each of experimental and control white pre-pupae were dissected and stained with neutral red (Fujioka et al. 2005) to detect PCs (see above). All 40 experimental animals ( $\mathrm{PC}-$ ) were completely devoid of PCs (Fig. 2d), whereas the control animals (PC+) had the normal range of 20-22 pairs of PCs (Fig. 2c). The remaining 60 animals of each group were allowed to develop into adults. Animals of both groups eclosed at similar times and were morphologically indistinguishable (except for the $\mathrm{CyO}$ wing phenotype in $\mathrm{PC}+$ ). Ten adults of each group were dissected within $12 \mathrm{~h}$ of eclosion and stained with neutral red. None of the animals of the PCgroup had any PCs (Fig. 2j). The PC+ group of adults showed the expected reduction in $\mathrm{PC}$ number after metamorphosis (Fig. 2g) as that reported for wild-types (Sellin et al. 2006). These results clearly demonstrate that an absence of post-embryonic PCs does not affect the viability of Drosophila adult flies under laboratory conditions.

Absence of pericardial cells does not affect cardiac remodelling during metamorphosis

The adult dorsal vessel (Fig. 2b) develops from the larval counterpart (Fig. 2a) following remodelling during pupation (Molina and Cripps 2001). The cardiac tube can be examined for defects in remodelling by staining for actin polymerization (by using Phalloidin) and by analysis of $\mathrm{CB}$ markers (Monier et al. 2005). To examine whether PCs are important for cardiac tube remodelling, we compared the 
Fig. 1 The srpHemo-GAL4 driver is active in PCs from second larval srpHemo-GAL4 $U A S-E G F P$ instars as shown by GFP expression (brackets anterior and posterior limits of dorsal vessel, $A E L$ after egg laying). a First larval instar immunostained for Pericardin $($ Prc, red $)$. b Second larval instar showing GFP expression (green). c Third larval instar (green GFP expression, asterisks lymph gland lobes, arrowheads haemocytes, white boundary outline of lymph glands and cardiac tube). Dorsal view with anterior to left. Bar $100 \mu \mathrm{m}$
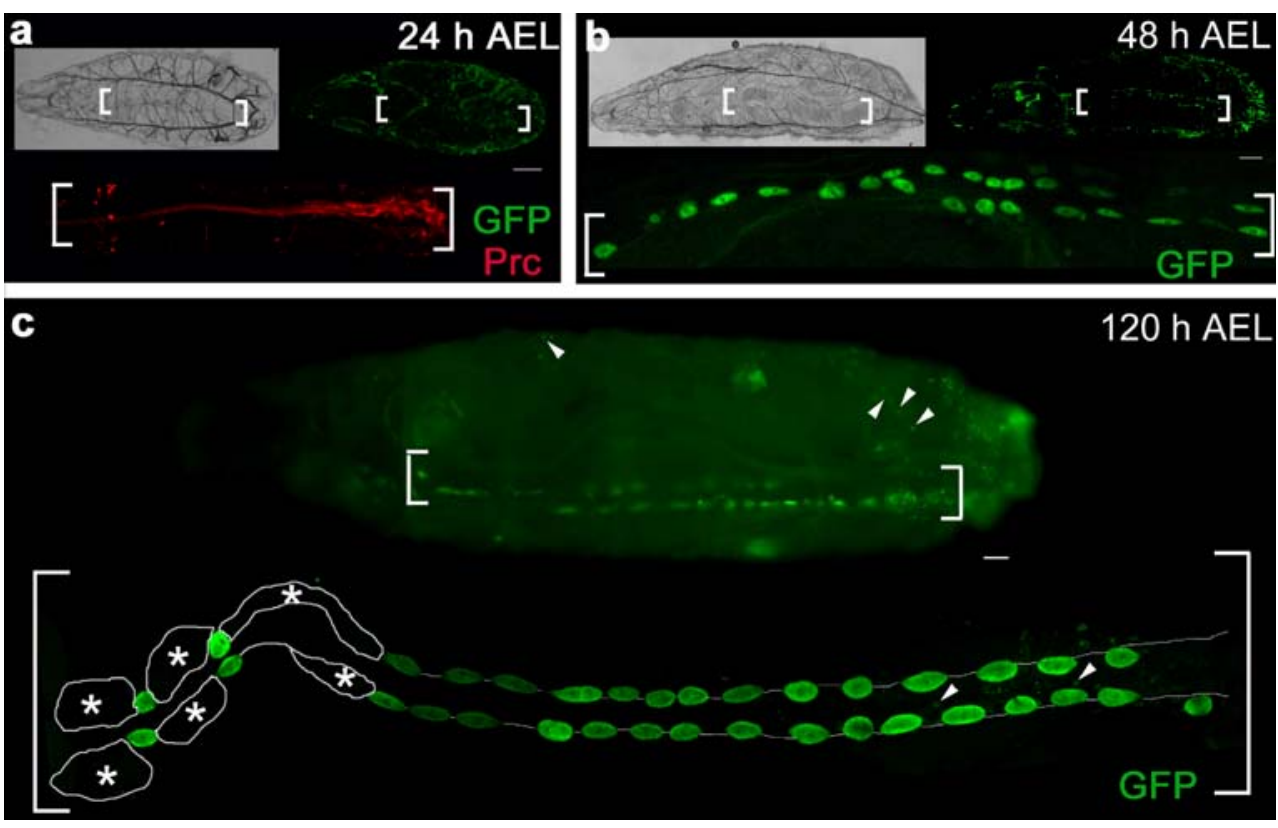

gene expression and morphology of the dorsal vessel of white pre-pupa and adult flies in animals with and without PCs. To retain tissues in their original context, we stained semi-dissected preparations in which the dorsal vessel was still attached to the abdomen. We observed that the expression of Tinman (Tin), a well-characterized marker for CBs (Cripps and Olson 2002) is not affected by loss of PCs (compare Fig. 2f, 1 with Fig. 2e, k, respectively). Comparable numbers of Tin-expressing cells were observed for both PC+ and PC- animals. Prc, an extracellular matrix protein that surrounds CBs and PCs, serves as a marker for the dorsal vessel. Whereas PCs, oenocytes and a subset of CBs express Prc in the embryo (Chartier et al. 2002), the source of post-embryonic Prc is not known. We observed Prc expression, even in the absence of PCs (compare Fig. 2h, n with Fig. $2 \mathrm{~g}, \mathrm{~m}$ ), which suggests that it may be persisting embryonic/early larval Prc and/or that other adult cells can also produce Prc in PC- animals. Staining for polymerized F-actin suggested that the overall morphology of the cardiac tube was similar between PC+ (Fig. 2o) and PC- (Fig. 2p) animals at this resolution. Since these animals had lost all PCs by the white pre-pupa stage, our result indicates that loss of PCs does not result in any gross aberration in cardiac remodelling during metamorphosis. Although this does not rule out the possibility of finer abnormalities being present, any such defect, if present, clearly does not affect the heartbeat rate of the animals (see below). All PC- white pre-pupae had one pair of primary and two to three secondary lymph gland lobes similar to that seen in PC+ and wild-type animals (Jung et al. 2005).
Loss of all pericardial cells does not affect heartbeat rate

Fujioka et al. (2005) report that a decrease in PC number through the loss of Eve expression in embryonic mesoderm reduces heart rate. To investigate whether ablation of postembryonic PCs affects cardiac function, we counted the heartbeat rate of all $\mathrm{PC}-$ and $\mathrm{PC}+$ white pre-pupae prior to dissection (see above). No significant difference $(P=0.8)$ was found between the heart rate of animals with or without PCs (Fig. 3a). This suggests that PC function is not required for maintaining normal cardiac rate under routine laboratory conditions. Thus, a decrease in the number of PCs cannot in itself explain the cardiac defects of eve meso animals observed by Fujioka et al. (2005). Certain aspects of dorsal vessel development might require the embryonic function of Eve in the mesoderm. Thus, a lack of mesodermal eve expression during embryogenesis could potentially affect cardiogenesis and cause phenotypes observed in the eve meso ${ }^{-}$animals. Our experimental strategy does not affect embryonic heart development and hence does not affect cardiac function.

\section{Absence of pericardial cells affects longevity}

PCs are a class of nephrocyte (Ward and Coulter 2000) that function to remove macromolecular waste from the haemolymph (Crossley 1985). Hence, their absence would lead to an accumulation of harmful molecules, thereby affecting homeostasis. However, our data suggest that this effect on homeostasis does not affect normal development. We 
Fig. 2 Drosophila adults develop without PCs (brackets anterior and posterior limits of dorsal vessel). Representations of white pre-pupal (a) and adult (b) dorsal vessel showing cardiac tube with cardiac nuclei (green) and PCs (brown). The abdominal segments (A1-A8) and thoracic segment (T3) are indicated. Hemi-dissected white pre-pupa (c-h) or adult (i-p) $\mathrm{PC}+$ or $\mathrm{PC}-$ animals (as indicated) stained with neutral red (c, d, i, j) or immunostained for Tinman (Tin; e, f, k, l; green) marking cardiac nuclei or for Prc $(\mathbf{g}, \mathbf{h}, \mathbf{m}, \mathbf{n} ;$ red) marking the extracellular matrix of the dorsal vessel or stained with phalloidin $(\mathbf{o}, \mathbf{p} ;$ red $)$ marking polymerized F-actin in the cardiac tube (arrowheads, hatch marks nonspecific staining in fat and muscle, solid white lines in $\mathbf{e}, \mathbf{f}$, $\mathbf{k}, \mathbf{I}$ limits of cardiac tube). Lymph gland boundaries are also marked with white dotted lines in $\mathbf{e}, \mathbf{f}$ and indicated by asterisks. Note the cardiac tube midline (dotted line in $\mathbf{g}, \mathbf{h}$, m-p). Dorsal view with anterior to left. Bar $100 \mu \mathrm{m}$ (a-j, m-p), $50 \mu \mathrm{m}(\mathbf{k}, \mathbf{l})$ a

a C

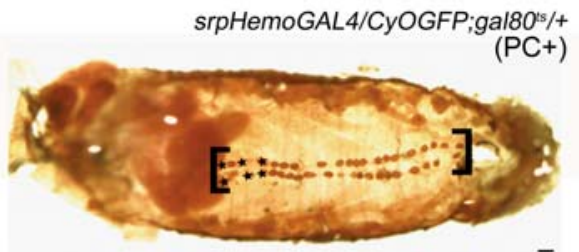
द. \begin{tabular}{l|l|l|l|l|l|l|l|l}
$\mathrm{T} 3$ & $\mathrm{~A} 1$ & $\mathrm{~A} 2$ & $\mathrm{~A} 3$ & $\mathrm{~A} 4$ & $\mathrm{~A} 5$ & $\mathrm{~A} 6$ & $\mathrm{~A} 7$ & $\mathrm{~A} 8$
\end{tabular} b

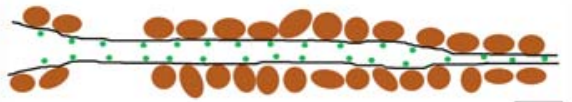
\begin{tabular}{l|l|l|l|l|} 
A1 & A2 & A3 & A4 & A5
\end{tabular}

d

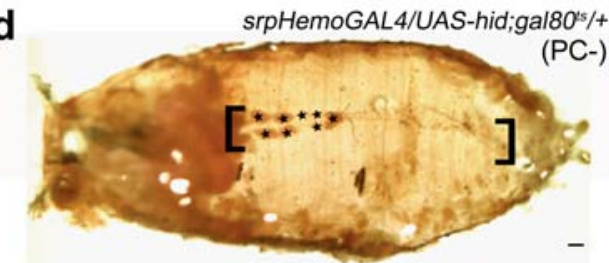

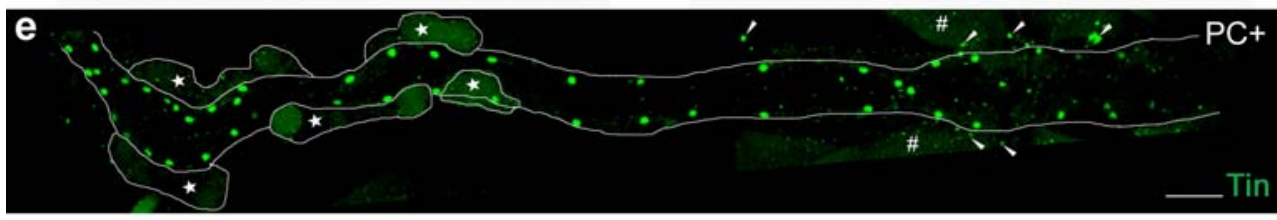
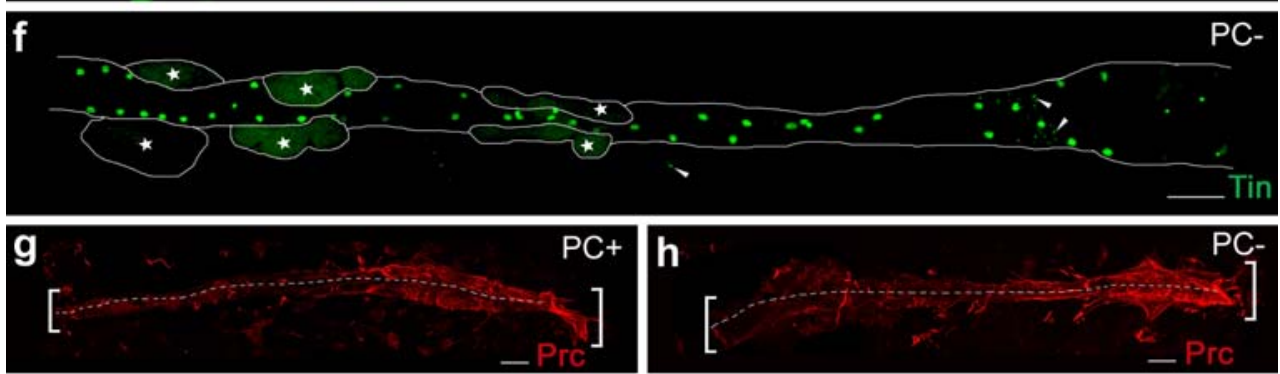

\section{i}

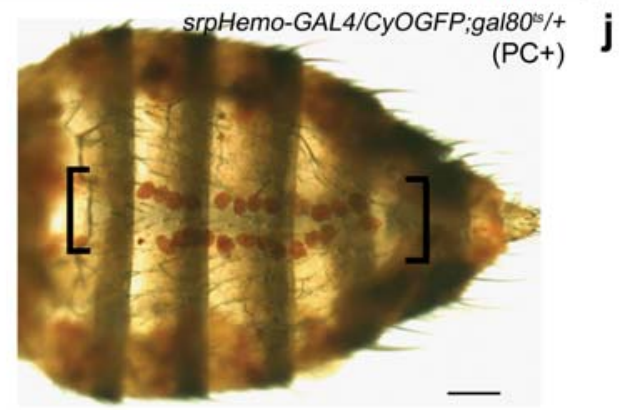

srpHemo-GAL4/UAS-hid;gal80ts/+

(PC-)
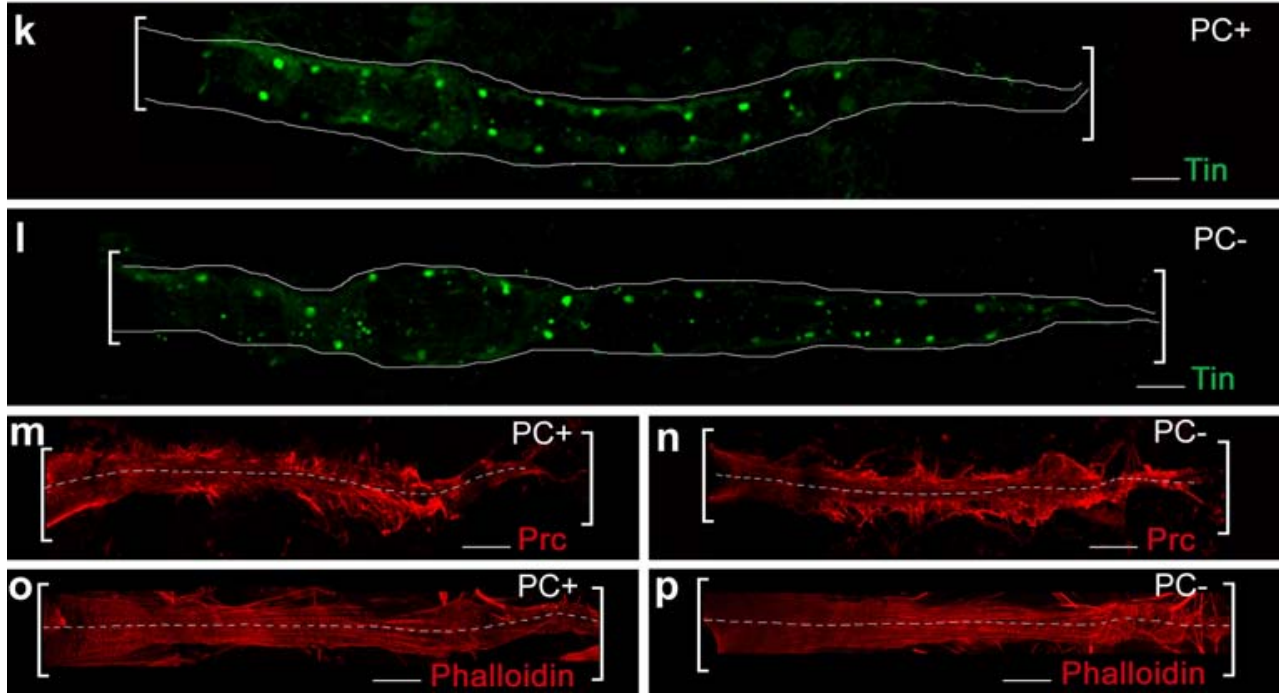

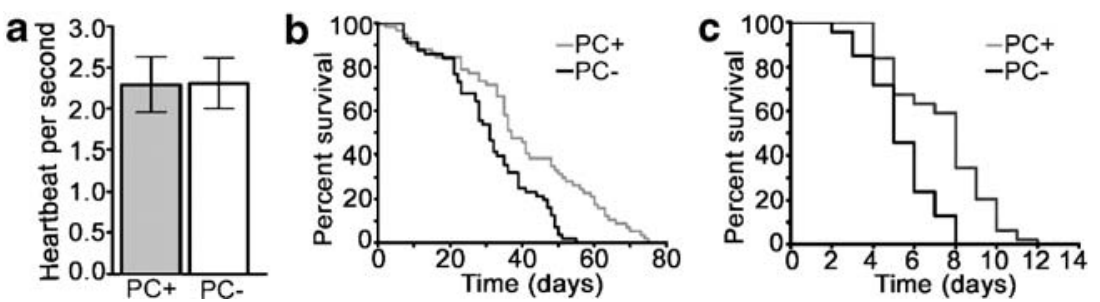

Fig. 3 Drosophila adults without PCs have reduced lifespan. a Graph of average heartbeat (beats/s) of white pre-pupae of indicated groups ( $n=40$; error bars standard deviation). b, c Graphs of lifespan

measurement of animals of indicated groups $(n=50)$ represented as percentage survival versus survival (in days). Animals were fed on normal food (b) or food with silver nitrate (c)

therefore examined whether PC removal could affect lifespan. For this, we compared the survival and lifespan of PCand $\mathrm{PC}+$ flies. For the first 20 days, no difference was seen in the death rate between $\mathrm{PC}-$ and $\mathrm{PC}+$ flies. Interestingly, after 20 days, the death rate increased in $\mathrm{PC}-$ flies, making the maximal life span significantly different $(P<0.05$, see Supplementary Methods) between these two groups (Fig. 3b). These data suggest that PCs are important for the survival of adult flies. What cellular role might PCs perform that requires their presence for a normal lifespan? Since PCs function in the segregation and storage of wastes from the haemolymph, their absence might result in an increased accumulation of metabolic waste products possibly leading to the early demise of PC- flies. The PC- flies that we have generated additionally lack a fraction of haemocytes that are still expressing Srp at the time of heat shock. Drosophila haemocytes have two origins: the embryonic mesoderm (embryonic haemocytes, EHs) and lymph gland (LGH). All haemocytes present in the larval stages are EHs, whereas the adult haemocyte population is composed predominantly of LGH and a small fraction of EHs that persist through metamorphosis (Holz et al. 2003). The srpHemo-GAL4 driver that we have used is active only in EHs but not in lymph glands (Fig. 1c, asterisk). Hence, the majority of adult haemocytes remain unaffected, even after the induction of Hid expression. This is evident from the presence of multilobed lymph glands in $\mathrm{PC}-$ animals (Fig. 2d, asterisk) similar to those of $\mathrm{PC}+$ animals (Fig. 2c, asterisk). The effect of EHs on survival has not been studied and the significance of the two separate populations of haemocytes remains unknown. The contribution of the partial loss of haemocytes to the survival/longevity phenotype seen upon ablation of PCs could be resolved by exclusively ablating PCs. However, as a PC-specific GAL4 driver is presently unavailable, this cannot be achieved.

To analyse the possible effect of PC loss on survival, we placed $\mathrm{PC}+$ and $\mathrm{PC}-$ flies under a stress condition in which $\mathrm{PC}$ function is absolutely essential. Since PCs also sequester toxic molecules (Mills and King 1965), we reasoned that their role would be more apparent in the presence of such molecules. Silver nitrate is a potent toxin (Bianchini et al. 2005), which, after ingestion, is released from the gut into the haemolymph and sequestered by PCs
(Crossley 1985). Therefore, we investigated the survival of $\mathrm{PC}-$ and $\mathrm{PC}+$ animals in the presence of silver nitrate. The survival of flies lacking PCs was significantly reduced $(P<0.001$, see Supplementary Methods) compared with that of control flies (Fig. 3c) in the presence of silver nitrate. This suggests that, since PCs can sequester toxic waste, they have a role in maintaining homeostasis and hence influence lifespan.

In spite of being the only cells accessory to the cardiac tube throughout development, surprisingly little is known about PC function. Using cell ablation, we show that PCs are not essential for adult development. However, their role in cardiac regulation is more complicated than that suggested earlier. PCs are required for normal cardiogenesis but have no effect on cardiac function post-embryogenesis. However, one can readily conceive possibilities of the way that developmental defects in the cardiac tube arising from a perturbation of embryonic PCs would affect cardiac function in post-embryonic stages. PCs are suggested to secrete cardio-regulatory molecules (Crossley 1985). For example, a serotonin-like indolylalkalamine identified in Periplanata americana has pharmacological effects on the heart (Crossley 1985). Our observation that animals without PCs have a normal cardiac rate clearly demonstrates that normal PC secretion does not have any cardio-regulatory function under laboratory conditions. However, uptake and sequestration by PCs are important for toxin clearance and this function may indirectly affect cardiac function in some situations.

Thus, our study has clarified unambiguously a number of speculations associated with PC function and has indicated a possible role of PCs in insect physiology, i.e. in toxin clearance and in the maintenance of homeostasis. In addition, the strategy that we describe here now provides the opportunity to generate animals with variable number of PCs at different developmental stages. This could be instrumental in addressing many unanswered questions regarding $\mathrm{PC}$ biology.

Acknowledgements We are grateful to Richard Cripps, Manfred Frasch, V. Sriram, Pernille Rorth, Helen Skaer and K. VijayRaghavan for antibodies, fly stocks and valuable comments, to B.S. Suma for help with confocal microscopy and to V. K. Sharma for advice on statistical analysis. 


\section{References}

Alvarez AD, Shi W, Wilson BA, Skeath JB (2003) Pannier and pointedP2 act sequentially to regulate Drosophila heart development. Development 130:3015-3026

Bianchini A, Playle RC, Wood CM, Walsh PJ (2005) Mechanism of acute silver toxicity in marine invertebrates. Aquat Toxicol 72:67-82

Bier E, Bodmer R (2004) Drosophila, an emerging model for cardiac disease. Gene 342:1-11

Brand AH, Perrimon N (1993) Targeted gene expression as a means of altering cell fates and generating dominant phenotypes. Development 118:401-415

Brodu V, Mugat B, Roignant JY, Lepesant JA, Antoniewski C (1999) Dual requirement for the EcR/USP nuclear receptor and the dGATAb factor in an ecdysone response in Drosophila melanogaster. Mol Cell Biol 19:5732-5742

Bruckner K, Kockel L, Duchek P, Luque CM, Rorth P, Perrimon N (2004) The PDGF/VEGF receptor controls blood cell survival in Drosophila. Dev Cell 7:73-84

Chartier A, Zaffran S, Astier M, Semeriva M, Gratecos D (2002) Pericardin, a Drosophila type IV collagen-like protein is involved in the morphogenesis and maintenance of the heart epithelium during dorsal ectoderm closure. Development 129:3241-3253

Cripps RM, Olson EN (2002) Control of cardiac development by an evolutionarily conserved transcriptional network. Dev Biol 246:14-28

Crossley A (1985) Nephrocytes and pericardial cells. In: Kerkut GA, Gilbert LI (eds) Comprehensive insect physiology, biochemistry and pharmacology. Pergamon, Oxford, pp 487-515

Fujioka M, Wessells RJ, Han Z, Liu J, Fitzgerald K, Yusibova GL, Zamora M, Ruiz-Lozano P, Bodmer R, Jaynes JB (2005) Embryonic even skipped-dependent muscle and heart cell fates are required for normal adult activity, heart function, and lifespan. Circ Res 97:1108-1114
Holz A, Bossinger B, Strasser T, Janning W, Klapper R (2003) The two origins of hemocytes in Drosophila. Development 130:4955-4962

Johnson AN, Burnett LA, Sellin J, Paululat A, Newfeld SJ (2007) Defective decapentaplegic signaling results in heart overgrowth and reduced cardiac output in Drosophila. Genetics 176:1609 1624

Jung SH, Evans CJ, Uemura C, Banerjee U (2005) The Drosophila lymph gland as a developmental model of hematopoiesis. Development 132:2521-2533

Mandal L, Banerjee U, Hartenstein V (2004) Evidence for a fruit fly hemangioblast and similarities between lymph-gland hematopoiesis in fruit fly and mammal aorta-gonadal-mesonephros mesoderm. Nat Genet 36:1019-1023

McGuire SE, Le PT, Osborn AJ, Matsumoto K, Davis RL (2003) Spatiotemporal rescue of memory dysfunction in Drosophila. Science 302:1765-1768

Mills RP, King RC (1965) The pericardial cells of Drosophila melanogaster. Q J Microsc Sci 106:261-268

Molina MR, Cripps RM (2001) Ostia, the inflow tracts of the Drosophila heart, develop from a genetically distinct subset of cardial cells. Mech Dev 109:51-59

Monier B, Astier M, Semeriva M, Perrin L (2005) Steroid-dependent modification of Hox function drives myocyte reprogramming in the Drosophila heart. Development 132:5283-5293

Sellin J, Albrecht S, Kolsch V, Paululat A (2006) Dynamics of heart differentiation, visualized utilizing heart enhancer elements of the Drosophila melanogaster bHLH transcription factor Hand. Gene Expr Patterns 6:360-375

Ward EJ, Coulter DE (2000) Odd-skipped is expressed in multiple tissues during Drosophila embryogenesis. Mech Dev 96:233236

Yi P, Han Z, Li X, Olson EN (2006) The mevalonate pathway controls heart formation in Drosophila by isoprenylation of Ggammal. Science 313:1301-1303 